\title{
Yohimbine intake-health risks - Correlation between side effects and the quality of the yohimbine bark: A UHPLC/MS, GC/MS screening.
}

\author{
Stanislava Ivanova ${ }^{1}$, Anna Mihaylova ${ }^{2}$, Penka Petleshkova ${ }^{3 *}$, Kalin Ivanov ${ }^{1}$ \\ ${ }^{1}$ Department of Pharmacognosy and Pharmaceutical Chemistry, Faculty of Pharmacy, Medical University of Plovdiv, \\ Plovdiv, Bulgaria \\ ${ }^{2}$ Medical College, Medical University of Plovdiv, Plovdiv, Bulgaria \\ ${ }^{3}$ Department of Obstetrics and Gynecology, Plovdiv Medical University, Plovdiv, Bulgaria
}

\begin{abstract}
After the intake of a yohimbine bark food supplement a thirty-eight-year old man declared serious complaints: headache, rapid heart rate, high blood pressure, cold sweating, rush, tremor. The patient took only 1 capsule per day every morning for two days. After the discontinuation of supplementation the symptoms disappeared. The purpose of the study was to determine the factors causing the described side effects. Series of analyses were performed: HPLC/MS and GC/MS. Yohimbine bark contains a number of different alkaloids. It was important to be established the content of the main alkaloidyohimbine because the content of yohimbine was not declared on the label. We also suspected the presence of undeclared sibutramine. The screening did not find presence of sibutramine but it was found that the total content of yohimbine in 1 capsule was $50 \mathrm{mg}$. $50 \mathrm{mg}$ of yohimbine per 1 capsule is extremely high dose- the approved daily dose of yohimbine in medicinal product is up to $30 \mathrm{mg} / \mathrm{person} /$ day. We also established presence of $\alpha$-yohimbine (ajmalicine) in high levels. We consider that the total content of yohimbine should be declared on the label of every food supplement which contains yohimbine bark, otherwise the customers would be exposed to risk.
\end{abstract}

Keywords: Yohimbine, Pausinystalia yohimbe, Yohimbine bark, Yohimbine, Food supplements, Safety, Non labelled substances, Yohimbine side effects.

Accepted on May 6, 2019

\section{Introduction}

Yohimbine is an indole alkaloid isolated from the bark of the African evergreen tree Pausinystalia yohimbe [1] (Figure 1). The bark is described as smooth and grey [1]. It contains a number of other alkaloids like: $\beta$-yohimbine, pseudoyohimbine, corynanthine, alloyohimbine and raubasine (also known as ajmalicine) [2,3]. Quantitative data on the chemical composition of the alkaloids in yohimbine bark is extremely variable. The chemical composition depends on factors such as growing and harvesting conditions of the plant. Usually the value of total alkaloid content of the bark ranges from about 2 to over $150 \mathrm{mg} / \mathrm{g}$. Yohimbine - the predominant alkaloid in the bark is an alpha-2- blocker $[1,4]$. It acts at the serotoninergic and adrenergic receptors in brain centres, associated with libido [3]. Compared to placebo, yohimbine intake increases diastolic blood pressure and plasma noradrenaline concentration [2]. Other serious adverse effects are also associated with yohimbine intake: dizziness, tremor, sweating, flushing, confusion, vomiting, anorexia, gastric complaints, nausea etc. [5]. Yohimbine hydrochloride is an active ingredient of a number of medicinal products for treatment of erectile dysfunction authorised in several EU countries. The doses vary from 5 to $10 \mathrm{mg}, 2 / 3$ times daily. The typical treatment period is about 8 weeks. Yohimbine is also available in numerous food supplements containing yohimbine bark. These products are promoted to improve sexual and athletic performance and are also promoted as weight loss products [2]. Yohimbine, can either be obtained from the bark or chemically synthesised

There is no information on standardisation of the extracts used for manufacturing of food supplements-starting material, extraction solvent, content of biologically active ingredients $[2]$.

In United Kingdom, Ireland, Netherlands, Belgium, Denmark, Czech Republic, Canada, Australia and New Zealand the inclusion of yohimbine bark and its preparations is specifically prohibited in foods or food supplements, because of the risk of serious adverse effects. In the most part of the World yohimbine containing products are labelled as food supplements. 
For identification of Yohimbine HCL European Pharmacopeia recommends [6]. Infrared absorption spectrophotometry; Reaction of chlorides, determination of $\mathrm{pH}$, determination of specific optical rotation: from +101.0 to +105.0 , HPLC for detection of related substances. Specified impurities for yohimbine HCL are: A, F, G. Analytical control of impurities in substances for pharmaceutical use must be done for: B, C, $\mathrm{D}, \mathrm{E}$ (Figure 2). The quality control for food supplements is not obligatory. Impurities B, C, D, E could persist in different concentration in some Yohimbine FS.

Recent studies have estimated that the exposure to yohimbine from food supplements intake could be higher than the approved daily dose of yohimbine in the medicinal products (up to $30 \mathrm{mg} /$ person/day).

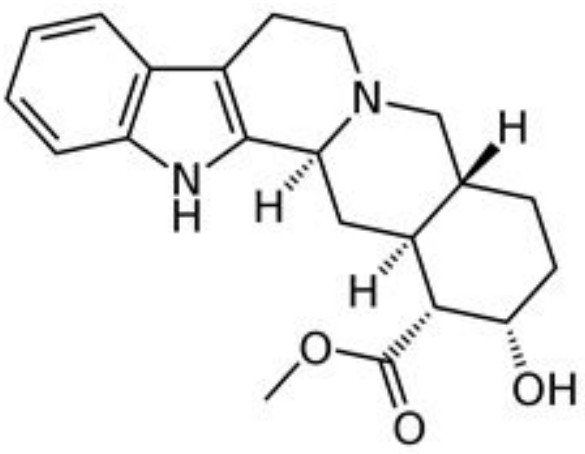

Figure 1. Yohimbine-structure.

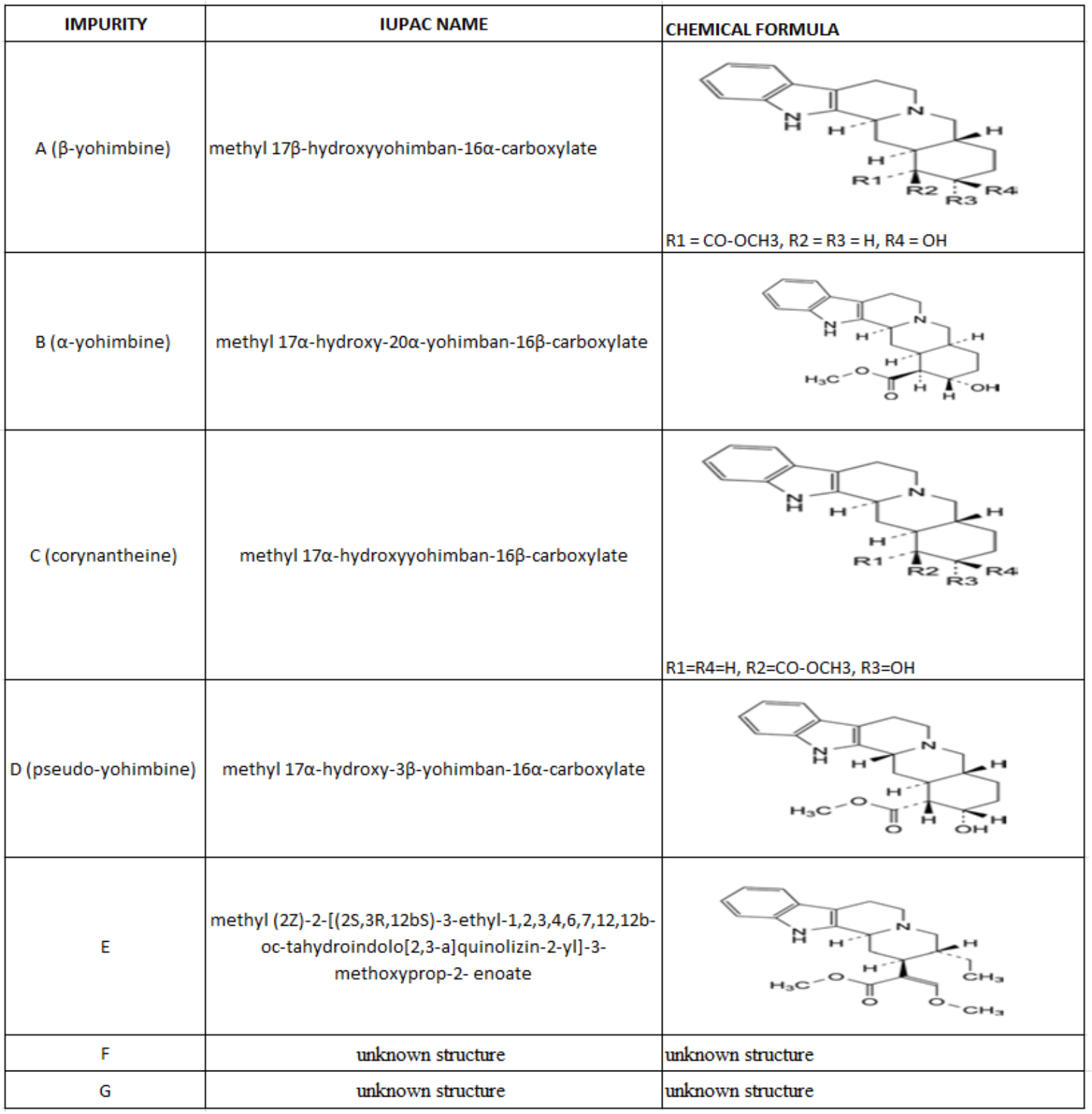

Figure 2. Yohimbine hydrochloride- impurities [6]. 


\section{Material and Methods}

\section{Materials}

Reference standards: Yohimbine hydrochloride with purity $>98 \%$, Sibutramine hydrochloride monohydrate with purity $>98 \%$.

Reagents with analytical grade: Methanol for HPLC $\geq 99.9 \%$ (Sigma Aldrich); Acetonitrile for HPLC $\geq 99.93 \%$ (Sigma Aldrich); Ammonium formate for HPLC $\geq 99.0 \%$ (Sigma Aldrich), Acetonitrile $\geq 99.9 \%$ (Sigma Aldrich) and distilled water.

Test sample: Capsules $500 \mathrm{mg}$. Content: Yohimbine barks 500 mg.

\section{Methods}

UHPLC/MS: "Accela UHPLC" with mass spectral detection HRMS "Q-Exative" with H-ESI- interface ("Thermo Fisher Scientific", Waltham, MA, USA) and columns "Kynetex" RP C8 $50 \times 3 \mathrm{~mm} \times 2.6 \mu \mathrm{m}$ type "core-shell" and "Poroshell" RP CE18 $150 \times 3 \mathrm{~mm} \times 2.7 \mu \mathrm{m}$ type "core-shell"; ultrasonic bath (Branson Wilmington, NC, USA); apparatus for ultra-pure water: "Milli-Q", "Milipore" (Bed-ford, MA, USA) and "Elga" (VWR International, Randor, PA, USA).

GH/MS: GC „Trace”, MS detector TSQ ("Thermo Fisher Scientific", Waltham, MA, USA), "split/splitless" injector, column: HP -35, $30 \mathrm{~m} \times 0.25 \mathrm{~mm} 0.25 \mu \mathrm{m}$ ("Phenomenex" Inc, Torrance, CA, USA)

\section{Results}

Current trend in obesity and overweight treatment is the intake of food supplements (FS). According recent studies the consumers of FS believe that these products are safe because they have mainly plant origin [7]. Labelling of a food supplement as "natural" does not mean that it is guaranteed not to have harmful adverse effects [4,8-25]. In most countries worldwide, food supplements are regulated as a food-category and this allows a very fast introduction on the market of new products. Data about the quality of the botanicals and the finished products are of major importance [8]. However the quality control, marketing and the sale of FS are not strictly regulated [26]. The aim of this study is a screening of a particular yohimbine supplement which caused serious side effects.

A thirty-eight years old man purchased from a pharmacy in the European Union a food supplement containing "yohimbine bark". The purpose of the supplementation was weight loss. Two hours after the first intake the patient came back to the pharmacy with serious complaints. The patient did not suffer from any chronic diseases. The patient was not obese. He reported that he does not take any other medicines or food supplements. Chief complaints included: headache, tremor, rush, sweating, anxiety, and discomfort. His heart rate was 110 beats/minutes; his blood pressure was $160 / 90 \mathrm{~mm} \mathrm{Hg}$. The Pharmacist recommended immediate discontinuation of the weight loss food supplement. The day after the patient felt much better and decided to continue the yohimbine intake, because he was not sure if the FS had evoked the symptoms. Soon after the intake of the food supplement he came back to the pharmacy with head ache, flush, tremor, high blood pressure (158/90) and heart rate 98 beats/ $\mathrm{min}$. The patient was directed for a medical examination. The physician recommended immediate discontinuation of the yohimbine intake because he concluded that the symptoms were associated with the particular food supplement. The manufacturer of the FS declared content of one capsuleyohimbine bark $500 \mathrm{mg}$. Dosage instructions - 1capsule, 2 times daily. The total quantity of yohimbine in 1 capsule was not declared. For screening of the product we have performed a series of analysis-UHPLC/MS and GC/MS. We have used previously validated analytical procedures [27].

To determinate the quantity of yohimbine we performed a HPLC/MS screening. It was performed gradient elution and isocratic elution in the next order:

Gradient: From 95\% mobile phase A/ 5\% mobile phase B to $95 \%$ mobile phase C/ $5 \%$ mobile phase B -10 min linear gradient.

Isocratic: $95 \%$ mobile phase $\mathrm{B} / 5 \%$ mobile phase $\mathrm{C}-15 \mathrm{~min}$;

Isocratic: $95 \%$ mobile phase $\mathrm{A} / 5 \%$ mobile phase $\mathrm{C}-5 \mathrm{~min}$.

Mobile phase A: water/ acetonitrile/ methanol (v/v/v 75:10:15); Mobile phase B: water/ acetonitrile/ methanol (v/v/v 50:30:20); Mobile phase C: $0.2 \mathrm{M}$ ammonium formate, $\mathrm{pH}=6.4$.

Preparation of the standard solution of Yohimbine HCL: $5.0 \mathrm{mg}$ of Yohimbine HCL- Sigma Aldrich was dissolved in 5 $\mathrm{ml}$ of mobile phase B and in $5 \mathrm{ml}$ of mobile phase A. From the basic standard solution by dilution with component $\mathrm{A}$ was obtained a working standard solution with concentration 100 $\mu \mathrm{g} / \mathrm{ml}$. Sample preparation: capsules were emptied and weighed. The content was extracted with the same solvents as the standard. All test solutions were filtrated twice. The first step included pre-filtration to remove larger particles from the solutions. For the finer filtration we used syringe filters (pore size $0.45 \mu \mathrm{m}$ ). The analyses were performed in positive ion mode with monitoring of the molecular ion of yohimbine with $\mathrm{m} / \mathrm{z}$ 355.20. We estimated that the total content of yohimbine in the analysed sample was $50 \mathrm{mg}$. per capsule. This high content of yohimbine definitely could be associated with some serious side effects like: anxiety, head ache, nausea, insomnia, irritability, increase of blood pressure and pulse rate, vomiting, anorexia, gastric complaints, flush, sweating, shivering, allergic reactions, nervousness, hypotension, tremor, bronchospasm [27] and others. Nonetheless another screening was performed. The purpose of the second screening was detection of non-labelled substances.

Many studies declared the adulteration of weight loss food supplements by synthetic therapeutic substances like sibutramine [27] - a drug prescribed for reduction of body mass in the recent past [27]. Sibutramine associated side effects 
corresponded to the condition of the patient. We supposed that the test sample could contain sibutramine. In order to detect sibutramine we used previously validated GC/MS method for detection of sibutramine: Gas chromatograph „Trace”, MS detector TSQ ("Thermo Fisher Scientific", Waltham, MA, USA), "split/split less" injector. A capillary column HP-35 was used as a stationary phase [30 $\mathrm{m}$ (length), $0.25 \mathrm{~mm}$ (diameter), $0.25 \mu \mathrm{m}$ (film)]. The temperature was programmed from $60^{\circ} \mathrm{C}$ to $325^{\circ} \mathrm{C}$. Carrier gas -helium. Data collection was performed over the mass range $50 \div 550 \mathrm{~m} / \mathrm{z}$. The identification of sibutramine was carried out by comparing its retention time and mass spectra with those of the reference standard. By dilution with acetonitrile it was prepared a standard solution of sibutramine $(\mathrm{c}=100 \mu \mathrm{g} / \mathrm{m})$. The injection volume was $1 \mu \mathrm{l}$. We established RT for sibutramine- 17, 4 minutes and quantifying ion $-114 \mathrm{~m} / \mathrm{z}$ (Figure 3). Sample preparation: capsules were emptied and weighed. The content was extracted with the same solvent as the standard. All test solutions were filtrated. We did not establish sibutramine in the test sample but we found a high level of ajmalicine (Figure 4) $(\mathrm{RT}=33.05)$. Ajmalicine is another alkaloid, naturally found in the bark. We considered that the serious condition of the patient was associated with the high dose of yohimbine.
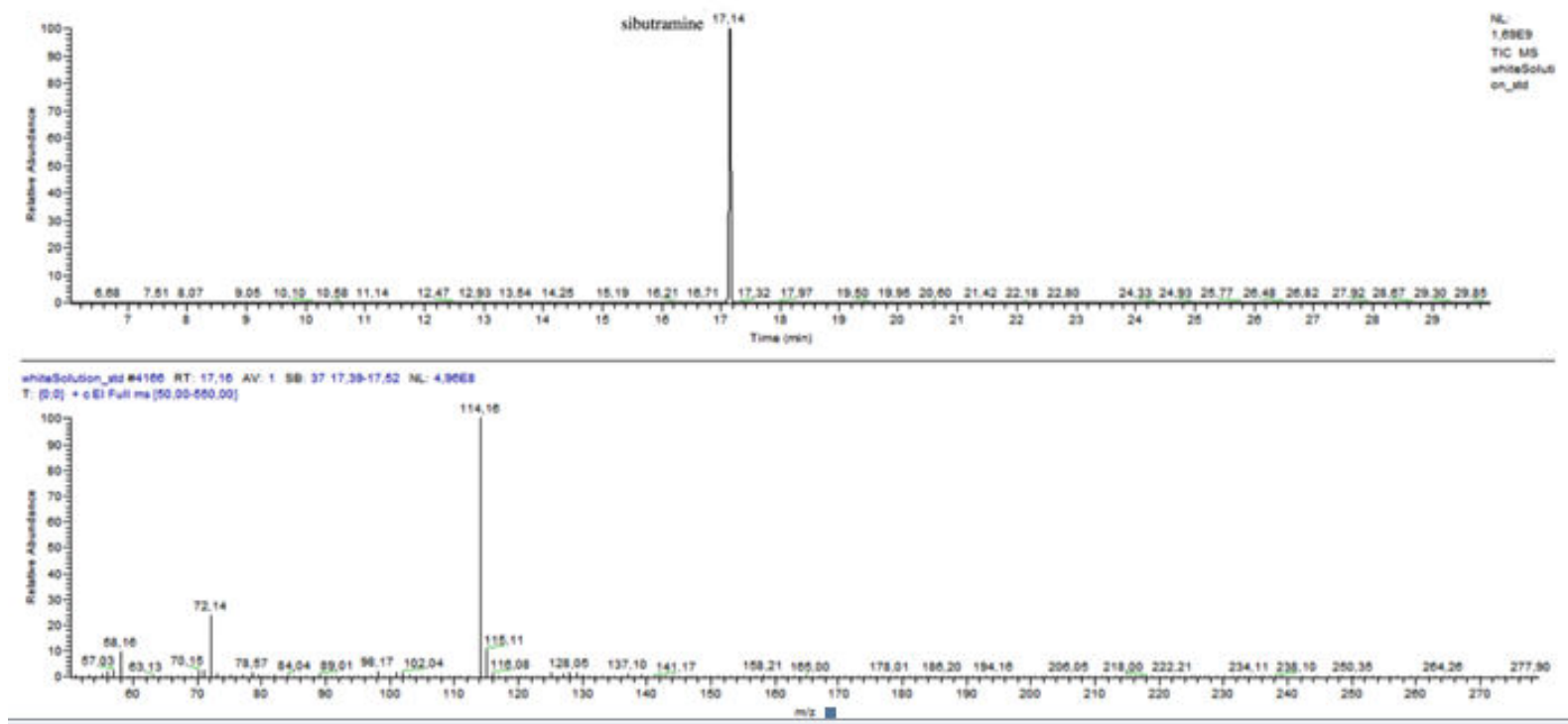

Figure 3. GC-MS chromatogram of sibutramine. The total ion chromatogram frame and the mass spectrum frame.

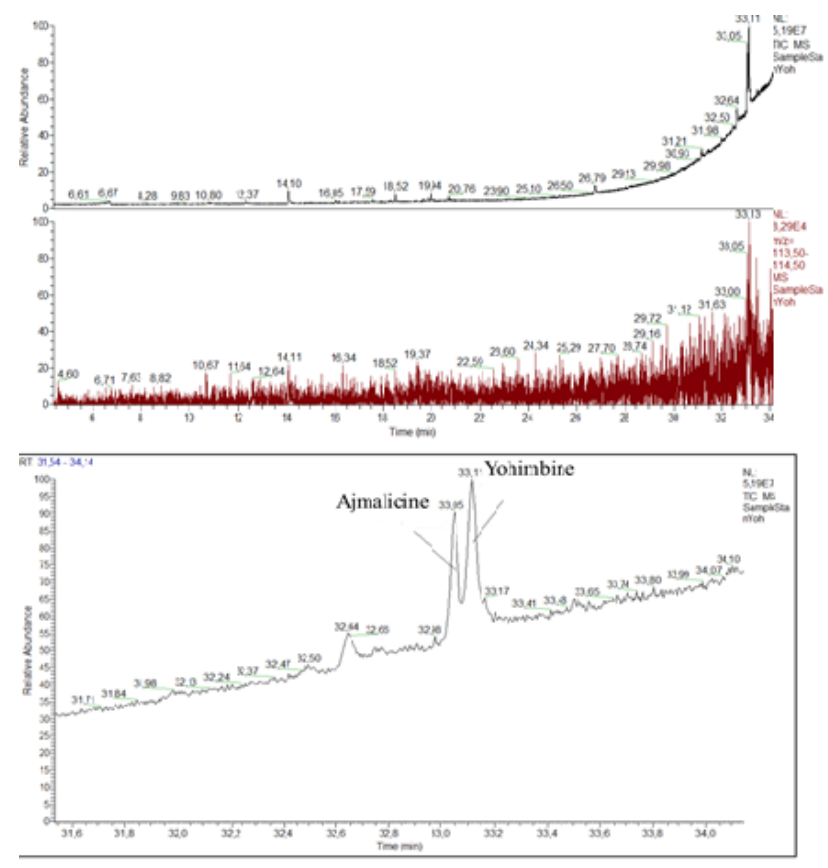

Figure 4. GC-MS chromatograms of the test sample.

\section{Conclusion}

We consider that supplementation with yohimbine bark could lead to serious side effects, because the total content of yohimbine in food supplements is not obligatory analysed and it could vary. We consider that the total content of yohimbine HCL should be declared on the label of every food supplement which contains yohimbine bark, otherwise the customers would be exposed to risk. The use of yohimbine or yohimbine bark products may result in serious side effects. Due to the high risk of adverse effects, it is important to consult with a physician before the intake of yohimbine containing products. We emphasise that it is extremely important that strategies for obligatory analytical control for food supplements should be developed.

\section{References}

1. Eweka AO, OmIniaboh FAE, Momodu O, "The histological effects of mixed diet containing Pausinystalia yohimbe ground stem bark on the kidney of adult Wistar rats (Rattus norvegicus)." Biol Med 2010; 2: 30-36.

2. EFSA Panel on Food Additives and Nutrient Sources Added to Food (ANS). "Scientific Opinion on the evaluation of the safety in use of Yohimbine 


\section{GC/MS screening}

( Pausinystalia yohimbe (K. Schum.) Pierre ex Beille)." EFSA Journal 2013; 11: 3302.

3. Tariq SH, Morley JE. Erectile Dysfunction, Encyclopedia of Neuroscience 2009; 1183-1189

4. Goldberg MR, Robertson D. Yohimbine: A pharmacological probe for study of the alpha 2adrenoreceptor. Pharmacol Rev 1983; 35: 143-180.

5. Tam, William S, Worcel M, Wyllie M. "Yohimbine: a clinical review." Pharmacology \& therapeutics. Pharmacol Ther. 2001; 91: 215-43.

6. European Pharmacopeia.

7. Khazan M, Hedayati M, Kobarfard F, Askari S, Azizi F. Identification and determination of synthetic pharmaceuticals as adulterants in eight common herbal weight loss supplements. Iran Red Crescent Med J 2014; 16: e15344.

8. Sanzini E, Badea M, Santos AD, Restani P, Sievers H "Quality control of plant food supplements."Food Funct 2011; 2: 740-746.

9. Pittler $\mathrm{MH}$, Schmidt $\mathrm{K}$ and Ernst E. Adverse events of herbal food supplements for body weight reduction: systematic review. Obes Rev 2005; 6: 93-111.

10. Carlsohn A, Cassel M, Linné K, Mayer F. How much is too much? A case report of nutritional supplement use of a high-performance athlete. $\mathrm{Br} \mathrm{J}$ Nutr 2011; 105: 1724-1728.

11. https://oig.hhs.gov/oei/reports/oei-01-01-00120.pdf.

12. Zou P. Detection of sibutramine, its two metabolites and one analogue in a herbal product for weight loss by liquid chromatography triple quadrupole mass spectrometry and time-of-flight mass spectrometry. Rapid Commun Mass Spectrom 2007; 21: 614-618.

13. Csupor D. Rapid identification of sibutramine in dietary supplements using a stepwise approach. Pharmazie 2013; 68: $15-18$.

14. Martello S, Marialinda F, Marcello C. Survey of nutritional supplements for selected illegal anabolic steroids and ephedrine using LC-MS/MS and GC-MS methods, respectively. Food Additives Contaminants 2007; 24: 258-265.

15. Lam $\mathrm{P}$, Leung $\mathrm{K}$, Wong $\mathrm{T}$, Lee $\mathrm{H}$, Tang $\mathrm{M}$, Mak $\mathrm{T}$, "Serotonin syndrome following overdose of a nonprescription slimming product containing sibutramine: a case report," Human and Experimental Toxicology 2012; 31: 414-417.

16. Kim H, Lee J, Park H, Cho S, Kim W, "Monitoring of 29 weight loss compounds in foods and dietary supplements by LC-MS/MS," Food Additives and Contaminants, Part A: Chemistry, Analysis, Control, Exposure and Risk Assessment 2014; 31: 77-783.

17. Gunaydin GP, Dogan NO, Levent S, Celik GK "Herbal weight loss pill overdose: sibutramine hidden in pepper pill." Case reports in emergency medicine 2015; 3.

18. Sanz JM, Sospedra I, Ortiz CM, Baladía E, Gil-Izquierdo A, Ortiz-Moncada R, Intended or Unintended Doping? A Review of the Presence of Doping Substances in Dietary Supplements Used in Sports. Nutrients 2017; 9: E1093. doi: 10.3390/nu9101093.

19. Maughan RJ. Quality assurance issues in the use of dietary supplements, with special reference to protein supplements. J. Nutr. 2013; 143: 1843S-1847S.

20. Thuyne VW, Eenoo VP, Delbeke FT, Nutritional supplements: Prevalence of use and contamination with doping agents. Nutr. Res. Rev. 2006; 19: 147-158.

21. Maughan RJ. Contamination of dietary supplements and positive drug tests in sport. J. Sports Sci. 2005; 23: 883889.

22. Pipe A, Ayotte C. Nutritional supplements and doping. Clin. J. Sport Med. Off. J. Can. Acad. Sport Med. 2002; 12: 245-249.

23. Green GA, Catlin DH, Starcevic B, Analysis of over-thecounter dietary supplements. Clin J. Sport Med. Off. J. Can. Acad. Sport Med. 2001; 11: 254-259.

24. https://www.fda.gov/drugs/resourcesforyou/consumers/ buyingusingmedicinesafely/ medicationhealthfraud/ ucm610148.html

25. Khazan M, Hedayati M, Kobarfard F, Askari S, Azizi F. Identification and determination of synthetic pharmaceuticals as adulterants in eight common herbal weight loss supplements. Iran Red Crescent Med J. 2014; 16: e15344.

26. Ivanova $\mathrm{S}$, Ivanov $\mathrm{K}$, Gueorgiev S,Petkova E. UHPLC/MS detection of yohimbine in food supplements. BMR 2017; 28: 3281-3285.

27. http://www.nobelmedicus.com/Content/1/22/100-102.pdf

\section{*Correspondence to}

Penka Petleshkova

Department of Obstetrics and Gynecology

Plovdiv Medical University

Bulgaria 